\title{
Liriodendrin alleviates sciatic endometriosis-associated pain in rats via suppressing inflammatory response and regulating the signaling pathway of $\mathrm{PI} 3 \mathrm{~K} / \mathrm{Akt} / \mathrm{mToR}$
}

\section{Type}

Research paper

\section{Keywords}

inflammation, pain, endometriosis, Akt, Liriodendrin, PI3K, mToR

\begin{abstract}
Introduction

LY294002 has been validated as a PI3K pan-inhibitor in the pathogenesis of airway inflammation, which attenuated IL-25-induced asthma-like AHR. Liriodendrin was proved to play essential roles in attenuating endometriosis-associated pain. This work aimed to gain a mechanical insight into the therapeutic efficiency of Liriodendrin administration on attenuating endometriosis-associated pain.

\section{Material and methods}

Von Frey filament test and thermal hyperalgesia test were carried out to evaluate the acute and daily efficiency of drug administration. Western blot was used to analyze the expression of substance $P$, $\mathrm{PI} 3 \mathrm{~K} / \mathrm{AKT} / \mathrm{mTOR}$, p-PI3K/p-AKT/p-mTOR, and CGRP. ELISA was performed to examine interleukin-1, interleukin-2, interleukin-6, TNF- $\alpha$ and PGE2 levels.
\end{abstract}

\section{Results}

As a result, Liriodendrin significantly attenuated pain in endometriosis rats and restored the upregulation of $\mathrm{p}-\mathrm{PI} 3 \mathrm{~K} / \mathrm{p}-\mathrm{AKT} / \mathrm{p}-\mathrm{mTOR}$ in the endometriosis rats. The increased interleukin-1, interleukin-2, interleukin-6, TNF- $\alpha$, and PGE2 levels were remarkably restored by Liriodendrin in endometriosis rats. Moreover, the activated expression of substance $\mathrm{P}$ in the ventral horn of the spinal cord of endometriosis rats was notably restored by Liriodendrin in addition to CGRP.

Furthermore, Liriodendrin effectively restored the LPS induced up-regulation of $p-P I 3 K / p-A K T / p-$ mTOR protein as well as the LPS activated IL-6, TNF- $\alpha$, and IL-1 $\beta$ mRNA and protein expression in $12 Z$ cells.

\section{Conclusions}

We found that compared with LY294200, Liriodendrin exerted a more evident therapeutic effect in the treatment of endometriosis-associated pain by suppressing the secretion of pro-inflammatory cytokines. 
Liriodendrin alleviates sciatic endometriosis-associated pain in rats via suppressing inflammatory response and regulating the signaling pathway of PI3K/Akt/mToR

3 Jianfang Gong ${ }^{1^{*}}$, Liangliang Xue ${ }^{1}$, Mengling Wei ${ }^{1}$, Wenli Han ${ }^{2}$, Shen Jing ${ }^{1}$

4 1. Department of Traditional Chinese Medicine, Shanxi Bethune Hospital, Taiyuan, Shanxi $5 \quad$ Province, China 030032

2. Department of Obstetrics and Gynecology, Shanxi Bethune Hospital, Taiyuan, Shanxi Province, China 030032

Corresponding author: Jianfang Gong, Department of Traditional Chinese Medicine, Shanxi Bethune Hospital, No. 99 Longcheng Street, Taiyuan City, Shanxi Province, China 030032

Email: fallotdr@yeah.net

\section{Abstract}

LY294002 has been validated as a PI3K pan-inhibitor in the pathogenesis of airway inflammation, which attenuated IL-25-induced asthma-like AHR. Liriodendrin was proved to play essential roles in attenuating endometriosis-associated pain. This work aimed to gain a mechanical insight into the therapeutic efficiency of Liriodendrin administration on attenuating endometriosisassociated pain. Von Frey filament test and thermal hyperalgesia test were carried out to evaluate the acute and daily efficiency of drug administration. Western blot was used to analyze the expression of substance $\mathrm{P}, \mathrm{PI}$ KK/AKT/mTOR, p-PI3K/p-AKT/p-mTOR, and CGRP. ELISA was performed to examine interleukin-1, interleukin-2, interleukin-6, TNF- $\alpha$ and PGE2 levels. As a result, Liriodendrin significantly attenuated pain in endometriosis rats and restored the upregulation of $\mathrm{p}-\mathrm{PI} 3 \mathrm{~K} / \mathrm{p}-\mathrm{AKT} / \mathrm{p}-\mathrm{mTOR}$ in the endometriosis rats. The increased interleukin-1, interleukin-2, interleukin-6, TNF- $\alpha$, and PGE2 levels were remarkably restored by Liriodendrin in endometriosis rats. Moreover, the activated expression of substance $\mathrm{P}$ in the ventral horn of the spinal cord of endometriosis rats was notably restored by Liriodendrin in addition to CGRP. Furthermore, Liriodendrin effectively restored the LPS induced up-regulation of $\mathrm{p}-\mathrm{PI} 3 \mathrm{~K} / \mathrm{p}-\mathrm{AKT} / \mathrm{p}$ mTOR protein as well as the LPS activated IL-6, TNF- $\alpha$, and IL-1 $\beta$ mRNA and protein expression in $12 Z$ cells. We found that compared with LY294200, Liriodendrin exerted a more evident 
therapeutic effect in the treatment of endometriosis-associated pain by suppressing the secretion of pro-inflammatory cytokines.

Running title: Liriodendrin alleviates endometriosis-associated pain in rats

Keywords: Liriodendrin, endometriosis, pain, inflammation, PI3K, Akt, mToR

\section{Introduction}

The presence of stromal or glandular cells on the edge of the uterine cavity is a defining characteristic of Endometriosis [1]. Chronic pain such as radicular pain, back pain, dyspareunia, and dysmenorrhea affect about $5 \%-10 \%$ of young women [2, 3]. Endometriosis affecting the sciatic nerve is a very rare condition [4]. The current treatment options include extracting the ectopic lesions and administration of hormones and NSAIDs [5]. Nonetheless, the current treatment options have limited efficacy and unwanted side effects [6].

Normal cellular functions such as metabolism, survival and growth are regulated by two signaling pathways: the phosphatidylinositol-3 kinases(PI3Ks) and the mammalian target of rapamycin (mTOR) $[7,8]$. Both the pathways are interconnected by a type of serine/threonine kinase-like AKT [9]. Protein kinase B also known as AKT plays a role in several cellular functions via PI3K and mTOR [10]. Stimuli from different types of receptors, such as antigen receptors, cytokine receptors, insulin receptors, or the insulin-like growth factor I receptor activate the PI3K and mTOR pathways. Upon activation, the PI3K causes phosphorylation of phosphorylates phosphatidylinositol 4,5-bisphosphate (PIP2) which produces a second messenger, phosphatidylinositol-3,4,5-trisphosphate (PIP3). PIP3 further stimulates downstream pathways like AKT. Elevated levels of PI3K/Akt/mTOR pathway were observed in the eutopic endometrium of women with endometriosis [11]. The multilevel signaling pathway involving PI3K, Akt and mTOR correlated well with pain and endometriosis [12].

51 Deying Dai, an expert in Chinese medicine, invented the prescription formula of Caulis 52 Sargentodoxae prescription. The formula was found to be effective against EMs (90\%), menstrual 53 fever (90.63\%) and dysmenorrhea (96.25\%) [13]. Extensive research has been performed to 
treating several inflammation conditions affecting the appendix, stomach and rheumatic arthritis. However, the mechanism of action of the medicine remains elusive. Another example of

57 traditional medicine called Liriodendrin, a type of lignin, has been studied for its therapeutic effects. This disaccharide lignan named Liriodendrin [(+)-syringaresinol di-O-b-Dglucopyranoside] is the bioactive component of Sargentodoxa cuneata (Oliv.) Rehd. Et Wils, and a plethora of biological effects against inflammation, pain, and arrhythmia were observed for the medicine [14, 15, 16]. This medicine has shown efficacy against disorders like arrhythmia, myocardial ischemia, inflammation and oxidative stress $[14,17]$. Moreover, according to previous findings, Liriodendrin administration restored the elevation of neuropeptides in the ventral horn of the spinal cord of ENDO rat models. Upon oral administration of Liriodendrin gets transformed to syringaresinol, which is attributable to the anti-inflammatory effect [17]. In various hormonedependent conditions, the phytoestrogen, Syringaresinol, binds selectively to the estrogen receptors [18].

The proposed mechanism of action of Liriodendrin involved reduced secretion of inflammationinducing cytokines like IL-6, TNF- $\alpha$ and IL-1 $\beta$ in the colon tissues. A similar decrease in proinflammatory cytokines was observed in DSS-induced colon damage models. In the intestine, a link was observed between the NF-KB cascade and the AKT signaling at transcription and translation levels $[19,20]$. Liriodendrin blocks protein-1 and/or NF-KB in SW982 human synovial sarcoma cells [21].

Based on the above-mentioned evidence, LY294002 may alleviate endometriosis-associated pain via regulating PI3K signaling, and Liriodendrin as an extract of Sargentodoxa Cuneata may also inhibit PI3K signaling, and in addition, Liriodendrin may also suppress the inflammatory response which is also involved in the endometriosis-associated pain. We aimed to validate our hypothesis that the administration of Liriodendrin could exhibit a therapeutic effect in the treatment of endometriosis-associated pain, the efficacy of which is supposed to be better than LY294002 in endometriosis. And the novelty of our study was to compare the efficacy of Liridodendrin and LY294002 in the treatment of endometriosis. 
Female SD rats with ages ranging between fourteen and eighteen weeks and weights in the range of 180-220 g were purchased from Charles River Laboratories Portage, MI. The rats were housed under alternating cycles of dark (12 h) and light (12 h) at room temperature and humidity near $70 \%$. The rats were allowed access to food and water. Animals were divided into four groups with 8 rats in each group, i.e., 1.) SHAM group (group of animals received the sham operation), 2.) ENDO group (group of animals were subjected to the surgical procedure and established as endometriosis rat model), 3.) ENDO+LY294002 group (endometriosis rat models subjected to the surgical procedure to create a rat model of endometriosis and received i.p. injection of LY294002) and 4.) ENDO+ Liriodendrin group (endometriosis rat models subjected to the surgical procedure to create a rat model of endometriosis and received oral administration of Liriodendrin). Models of sciatic nerve endometriosis were created using previously reported procedures [22]. Irrespective of the estrous cycle of the female rats, the autologous uterine tissue was grafted near the site of the sciatic nerve. Initially, the rats were put under anesthesia using isoflurane then a $2 \mathrm{~cm}$ long incision was created near the lower abdomen which exposed the right uterine horn. Later, a $5 \mathrm{~mm}$ long uterus tissue was dissected and extracted from the horn region of the uterus. It was kept in a Petri dish which was preloaded with a phosphate buffer solution containing 1\% Penicillin and Streptomycin. Subsequently, the right sciatic nerve was revealed on the rats positioned left side and an incision of $1 \mathrm{~cm}$ long near the thigh was made. The connective tissues near the desired area were cautiously separated. The longitudinal section of the uterus was opened and tied around the nerve with uterus endometrial tissue next to the nerve. In the case of rats that received the Sham operations, no implant was installed near the exposed right sciatic nerve. After the surgical procedure, 6.0-grade silk thread was used to suture the incision, and to prevent the infection gentamicin $(50 \mathrm{mg} / \mathrm{mL}, 0.2 \mathrm{~mL}$, Thermo Fisher Scientific, Waltham, MA) was injected into the stomach. In group 3, the dose of LY294002 was $15 \mathrm{mg} / \mathrm{mL}$ which was dissolved in DMSO. A dose of $30 \mathrm{mg} / \mathrm{kg}$ was administered to the animals via microsyringe into the i.t. tubing. In group 4, $100 \mathrm{mg} / \mathrm{kg}$ liriodendrin, was pretreated about 3 days before the surgery. The animal experiment procedures were approved by the institutional ethical committee.

\section{Behavioral testing}


112 To assess the behavior of the rats, Von Frey filament test was used. Briefly, the Von Frey filament 113 test was performed following the standard protocol which involves applying mechanical stimuli 114 to measure the hypersensitivity to mechanical stress. The key factor to measure was the paw withdrawal threshold. A series of ten Von Frey filaments of equal logarithmic bending force was used in a sequence. To perform the test, the rats were allowed to habituate on the wire gauge surface for $15 \mathrm{~min}$. To analyze the thermal hyperalgesia, a heat source was applied to the hind paw of the rats. The heat source was made up of a lamp fitted with the high-intensity bulb of power $50 \mathrm{~W}$, and a voltage of $8 \mathrm{~V}$. The exposure limit was decided to be $20 \mathrm{~s}$ so that the rats did not get skin burns. Following these behavior tests, the effects of LY294002 following the sciatic endometriosis procedure.

\section{Procedure for Abdominal Puncture}

123 The medicine was administered orally to rats for 21 days and after that $10 \mathrm{~mL}$ of PBS buffer was 124 filled into the stomach cavity of rats under anesthesia with the help of a syringe. The rats were shaken gently for 10 min and a puncture was made into the abdominal wall of the rats using a needle. The peritoneal fluid was extracted with the help of a needle. Later, the rats were euthanized, and the samples were collected. The upper clear layer of the peritoneal fluid and blood samples were obtained and refrigerated at $-80^{\circ} \mathrm{C}$.

\section{Enzyme-Linked Immunosorbent Assay}

The peritoneal fluid samples and blood samples were extracted and stored at $2-8{ }^{\circ} \mathrm{C}$ for a maximum of 5 days. Then, the substance $P$, interleukin-1, interleukin-2, interleukin-6, TNF- $\alpha$, PGE2, and CGRP levels were analyzed using the protocol provided with the kit in accordance with published methods [23]. The antibody was incubated and procedures for washing were performed based on the published methods. The absorbance of the ELISA experiments was recorded using the absorbance microplate reader (Molecular Devices, San Jose, CA).

\section{Cell Culture}

137 In this study, we established our cell models with $12 Z$ cells (Applied Biological Materials (abm) 
cultured in $37^{\circ} \mathrm{C}$ and $5 \% \mathrm{CO}_{2}$ in Roswell Park Memorial Institute 1640 medium containing $10 \mathrm{mM}$ HEPES and other antibiotics according to previously published methods [24]. The cells were transferred into 6-well plates and cultured until they were confluent. To these cells, the drugs at the desired concentration were added to the wells. The cells were divided into four groups, 1) Negative Control group, 2) LPS group; the cells were stimulated with the $100 \mathrm{ng} / \mathrm{mL}$ of lipopolysaccharide, 3) LPS+ LY294002 group; the cells were treated initially with 10 uM of LY294002 and six hours later with $100 \mathrm{ng} / \mathrm{mL}$ of LPS, and 4) LPS+ Liriodendrin group, the cells were pretreated with $50 \mathrm{uM}$ Liriodendrin and six hours later treated with $100 \mathrm{ng} / \mathrm{mL}$ of LPS. All experimental procedures were done four times using the above-mentioned protocol.

\section{RNA isolation and real-time PCR}

The authors performed a polymerase chain reaction test using the RNA extraction kit obtained from Thermo Fisher Scientific. The genetic material was removed from the samples using the kit. The reverse transcription of the RNA material in the samples into the cDNA was carried out using iScript $^{\mathrm{TM}}$ cDNA Synthesis Kit (BioRad, Hercules, CA). Later, the quantitative RT-PCR was carried out using the iTaq Universal SYBR Green Supermix (BioRad, Hercules, CA). The polymerase reaction was performed with Applied Biosystems Real-Time PCR Instruments (Thermo Fisher Scientific, Waltham, MA). The relative expression of substance $P$, interleukin-6, TNF- $\alpha$, interleukin-1 $\beta$, NF-KB and CGRP were calculated relative to the reference standard genes by the $2^{\Delta \Delta \mathrm{Ct}}$ method following published protocols [25].

\section{Western blot analysis}

The Western blot analysis was performed according to published protocols [26]. The cultured cells and tissue samples obtained from the decapitated animals were first washed with PBS buffer. Radioimmunoprecipitation buffer was used as a lysed buffer to treat the cells and tissues. This procedure was used to extract the enclosed proteins from the cells and tissue samples. The mixture of proteins obtained from the samples was separated into bands using $10 \%$ SDSpolyacrylamide gel. Then, the total protein content isolated from the cells was resolved on a $10 \%$ sodium dodecyl sulfate-polyacrylamide gel using an electrophoresis system (VWR, Radnor, PA). Later, the separated bands were expanded on a PVDF membrane and the reaction was blocked 
167 by skim milk. Subsequently, antibodies for CGRP (Cat\#ab47207, Abcam, Cambridge, UK), PI3K 168 (Cat\#ab32089, Abcam, Cambridge, UK), AKT(Cat\#ab8805, Abcam, Cambridge, UK), mTOR 169 (Cat\#ab134903, Abcam, Cambridge, UK), p-PI3K (Cat\#ab278545, Abcam, Cambridge, UK), p-AKT 170 (Cat\#ab38449, Abcam, Cambridge, UK), p-mTOR (Cat\#ab109268, Abcam, Cambridge, UK), 171 substance P (Cat\#ab14184, Abcam, Cambridge, UK), TNF- $\alpha$ (Cat\#ab183218, Abcam, Cambridge,

172 UK) and interleukin-1及 (Cat\#ab254360, Abcam, Cambridge, UK) were applied to the protein 173 bands followed by the secondary antibodies.

\section{Statistical analysis}

Statistical analysis was carried out by making use of SPSS 19.0 software (IBM, Chicago, IL). Oneway ANOVA and Student's t-tests were used to compare intergroup differences. Tukey's test was used as the post hoc test following one-way ANOVA. All statistical tests were two-sided, while the $p$-values of $<0.05$ indicated statistically significance.

\section{Results}

LY294002 or Liriodendrin administration attenuated the increased pain in endometriosis rat models

The paw withdrawal threshold was significantly decreased in ENDO rats when compared with the control. LY294002 and Liriodendrin administration remarkably maintained the paw withdrawal threshold in EODO treated rats, indicating the efficiency of acute drug administration (Fig.1A). Moreover, daily drug administration efficiency was evaluated every three days using the Von Frey filament test. LY294002 and Liriodendrin administration showed considerable efficiency in restoring the reduction in paw withdrawal threshold in the ENDO group(Fig.1B). Besides, a thermal hyperalgesia test was used to examine the efficiency of acute and chronic drug administration. LY294002 and Liriodendrin administration showed considerable efficiency in restoring the paw withdrawal latency loss in rats from the ENDO group in both acute drug administration (Fig.2A) and daily drug administration (Fig.2B) efficiency evaluation. 
The expression of PI3K/AKT/mTOR and p-PI3K/p-AKT/p-mTOR in the endometrium of rat groups was investigated (Fig.3A). No obvious difference was observed for the expression of PI3K (Fig.3B), AKT (Fig.3C) and mTOR (Fig.3D) in the endometrium of rats under distinct treatments. However, the expression of p-PI3K (Fig.3E), p-AKT (Fig.3F) and p-mTOR (Fig.3G) were remarkably increased in the endometrium of ENDO rats when compared with the control. LY294002 and Liriodendrin administration notably decreased the up-regulation of p-PI3K (Fig.3E), p-AKT (Fig.3F) and p-mTOR (Fig.3G) in the endometrium of rats with endometriosis.

\section{Liriodendrin administration restored the elevated of interleukin-1, interleukin-2, interleukin-6,} TNF- $\alpha$ and PGE2 in rats with endometriosis

The interleukin-1, interleukin-2, interleukin-6, TNF- $\alpha$ and PGE2 levels in the peritoneal fluid of rats with endometriosis were significantly increased when compared with the control. LY294002 and Liriodendrin administration notably decreased the elevation of interleukin-1, interleukin-2, interleukin-6, TNF- $\alpha$ and PGE2 in the peritoneal fluid of rats with endometriosis. Moreover, the therapeutic efficiency of Liriodendrin administration at the dose investigated was stronger than LY294002 administration at the dose investigated in restoring the levels of interleukin-1 (Fig.4A), interleukin-2 (Fig.4B), interleukin-6 (Fig.4C), TNF- $\alpha$ (Fig.4D) and PGE2 (Fig.4E) in the peritoneal fluid of rats. Furthermore, the PGE2 levels in the blood of rats were examined under distinct conditions. The serum level of PGE2 in ENDO rats was significantly increased when compared with the control. Liriodendrin administration notably decreased the elevation of PGE2 in the serum of the ENDO group, but no therapeutic efficiency was observed for LY294002 on restoring the PGE2 levels in the blood of rats from the ENDO group (Fig.4F).

\section{Liriodendrin administration restored the elevation of CGRP and substance P in the ventral horn} of the spinal cord of rats with endometriosis

LY294002 and Liriodendrin administration notably decreased the up-regulated substance P (Fig.5A) and CGRP level (Fig.5B) in the ENDO group. Moreover, LY294002 and Liriodendrin administration notably decreased the up-regulated substance P (Fig.5C, F, G) and CGRP (Fig.5D, $\mathrm{F}, \mathrm{H}) \mathrm{mRNA}$ and protein in the ventral horn of the spinal cord in the ENDO rats. It's worth noting that the efficiency of Liriodendrin administration at the dose investigated administration was 
222 more apparent than LY294002 administration at the dose investigated on maintaining the expression of CGRP and substance $P$ in the ventral horn of the spinal cord. And NF-kB mRNA level

224 (Fig.5E) showed same tendency as the gene expression level of CGRP and substance P.

225

226

227

228

229

230

231

232

233

234

235

236

237

238

239

240

241

242

243

244

245

246

247

248

LY294002 and Liriodendrin administration restored the LPS induced up-regulation of $\mathrm{p}-\mathrm{PI} \mathrm{KK} / \mathrm{p}$ AKT/p-mTOR in $12 Z$ cells.

The expression of PI3K/AKT/mTOR and p-PI3K/p-AKT/p-mTOR in $12 Z$ cells treated with LPS followed by LY294002 and Liriodendrin administration was investigated (Fig.6A). No obvious difference was observed for the expression of PI3K (Fig.6B), AKT (Fig.6C) and mTOR (Fig.6D) in $12 Z$ cells under differential conditions. However, the expression of p-PI3K (Fig.6E), p-AKT (Fig.6F) and p-mTOR (Fig.6G) were remarkably increased in LPS treated $12 Z$ cells when compared with the control. LY294002 and Liriodendrin administration notably decreased the LPS induced upregulation of p-PI3K (Fig.6E), p-AKT (Fig.6F) and p-mTOR (Fig.6G) in LPS treated $12 Z$ cells.

Liriodendrin administration restored the LPS induced up-regulation of IL-6, TNF- $\alpha$, and IL-1 $\beta$ mRNA and protein expression in $12 Z$ cells.

Moreover, Liriodendrin administration at the dose investigated showed stronger efficiency than LY294002 administration at the dose investigated on restoring the LPS induced up-regulation of IL-6 (Fig.7A), TNF- $\alpha$ (Fig.7B), and IL-1 $\beta$ (Fig.7C) mRNA in $12 Z$ cells. And Liriodendrin administration at the dose investigated showed more apparent efficiency than LY294002 administration at the dose investigated on restoring the LPS induced up-regulation of IL-6 (Fig.7E), TNF- $\alpha$ protein (Fig.7F), and IL-1 $\beta$ protein (Fig.7G) in $12 Z$ cells. And NF-KB mRNA level (Fig.7D) showed same tendency as the gene expression level of CGRP and substance P.

\section{Discussion}

A disaccharide lignan named Liriodendrin [(+)-syringaresinol di-O-b-Dglucopyranoside] is the bioactive component of Sargentodoxa cuneata (Oliv.) Rehd. Et Wils. A plethora of biological effects against inflammation, pain, and arrythmia was observed for the medicine [14, 15, 27]. In this study, we evaluated the efficiency of acute drug administration and daily drug administration of Liriodendrin on attenuating pain in endometriosis rats. Liriodendrin has been reported to 
suppress inflammatory damage in the treatment of ulcerative colit and sepsis-associated tissue injuries $[28,29]$. However, its effect in the treatment of endometriosis-associated pain was not investigated. Therefore, we aimed to explore and compare its effect in the treatment of endometriosis-associated pain with LY294002 in our study. As a result, we found that, despite both LY294002 and Liriodendirn were found to function as PI3K inhibitors, only Liriodendrin was found to alleviate inflammation, thus making Liriodendrin a better therapeutic method compared with LY294002. In addition, the authors compared the distinct expression of neuropeptides mRNA and protein in the ventral horn of spinal cord of ENDO rats. Liriodendrin administration restored the elevation of neuropeptides in the ventral horn of spinal cord of ENDO rats. Upon oral administration of Liriodendrin gets transformed to syringaresinol, which is attributed to the anti-inflammatory effect [15]. In various hormone-dependent conditions, the phytoestrogen, Syringaresinol, binds selectively to estrogen receptors [21].

Previous studies have shed light on the function of the mTOR/AKT pathway in endometriosis. One of the studies revealed that the P3KCA mutation, which is important for Akt activation, is frequently observed in patients with ovarian clear cell carcinoma [30,31]. Higher AKT activity has been observed in ovarian endometriosis compared to the normal endometrium, which could be possibly due to the estrogen in endometriotic cells [32]. The authors found that DIE patients showed higher activity of AKT in endometriotic lesions similar to ovarian endometriosis. A PI3K inhibitor, LY294002, was showed to inhibit the PI3K/Akt/mTOR signaling pathway in animals. In order to investigate the acute and chronic effects of intrathecal injection of LY294002 on various signaling pathways in sciatic nerve endometriosis models in rats including PI3K/Akt/mTOR. The effects of LY294002 in the animal models began at 2-3 h, peak effect was seen at four to five

271 hours and was four hours long. Daily injections of LY294002 for 3 weeks greatly reduced 272 mechanical and thermal hyperalgesia caused by sciatic nerve endometriosis (Fig.1 and Fig.2). The 273 therapeutic effects of LY294002 in sciatic nerve endometriosis models might be related to reduced expression levels of PI3K/Akt/mTOR signaling pathway-related factors (Fig.3). The 275 authors performed western blot analysis to determine the expression of the signaling proteins 276 and their phosphorylated counterparts in ENDO treated rats and LPS treated $12 Z$ cells. Treatment 277 with the drugs, LY294002 and Liriodendrin, reestablished the lower levels of phosphorylated 
278 signaling proteins in the animal and cellular models. In addition, the ELISA assay was performed 279 to measure the interleukin-1, interleukin-2, interleukin-6, TNF- $\alpha$ and PGE2 levels in stomach fluid 280 of ENDO treated rats. Previously, it was reported that injection of LY294002 significantly reduced 281 lung inflammation and airway hyperresponsiveness in an asthma animal model [33, 34].

282 Even though the mechanism is still unclear, recent studies have shown that swelling and modified 283 nerves in the endometriotic lesions are connected to the pathology of endometriosis. The 284 aberrant stimulation causes modification in neurotransmitter levels and also abnormal secretion 285 of inflammatory factors. The PI3K inhibitors reduced the levels of IL-6, MCP-1 (known also as 286 CCL2), TNFa and nitric oxide, which are the key factors regulating the inflammation process. The $287 \mathrm{PI3K} /$ Akt signaling pathway has been shown to regulate the NF-jB activation and phosphorylation 288 of p65 units of NF-jB [35]. In this study, we analyzed the differential expression of IL-6, TNF- $\alpha$, 289 and IL-1 $\beta$ in LPS treated $12 Z$ cells. Liriodendrin administration restored the LPS induced elevation 290 of IL-6, TNF- $\alpha$, and IL-1 $\beta$ in LPS treated $12 Z$ cells. The (+)-syringaresinol, produced in situ from 291 Liriodendrin by bacteria in intestines, enhanced FOXO3 and SIRT1 activity which further modulate 292 the activity of HIF-1 [36-38]. Hence, the authors hypothesized that Liriodendrin degrades HIF-1 $\alpha$ 293 by FOXO3 and SIRT1 activation, in turn reducing VEGF expression.

\section{Conclusion}

295 As a conclusion, we found that compared with LY294200, the administration exerted a more 296 evident therapeutic effect in the treatment of endometriosis-associated pain by suppressing the 297 secretion of pro-inflammatory cytokines.

298 Conflict of interest

$299 \quad$ None

$300 \quad$ Funding

$301 \quad$ None

302 Availability of data and material 
303 The data that support the findings of this study are available from the corresponding author upon

304 reasonable request.

\section{Author contributions}

306 JFG and LLX planned the study, collected the pieces of literature and analyzed the data, MLW,

307 WLH, SJ and JFG composed the manuscript and approved the final manuscript.

308 Figure legends

$309 \quad$ Fig.1

310 Chemical structure of Liriodendrin and the efficiency of acute/daily drug administration

311 evaluated by Von Frey filament test ( ${ }^{*}$ P value $<0.05$ vs. SHAM group; \# P value $<0.05$ vs. ENDO 312 group).

313 A: Acute LY294002 and Liriodendrin administration remarkably maintained the paw withdrawal 314 threshold in EODO treated rats.

315 B: Daily LY294002 and Liriodendrin administration remarkably maintained the paw withdrawal 316 threshold in EODO treated rats.

$317 \quad$ Fig.2

318 Acute/daily drug administration efficiency evaluation using Thermal hyperalgesia test (* $\mathrm{P}$ value $319<0.05$ vs. SHAM group; \# P value < 0.05 vs. ENDO group).

320 A: Acute LY294002 and Liriodendrin administration remarkably maintained the paw withdrawal 321 latency in EODO treated rats.

322 B: Daily LY294002 and Liriodendrin administration remarkably maintained the paw withdrawal 323 latency in EODO treated rats.

324 Fig.2

325 Acute/daily drug administration efficiency evaluated using Thermal hyperalgesia test (* $\mathrm{P}$ value $326<0.05$ vs. SHAM group; \# P value < 0.05 vs. ENDO group). 
327 A: Acute LY294002 and Liriodendrin administration remarkably maintained the paw withdrawal 328 latency in EODO treated rats.

329 B: Daily LY294002 and Liriodendrin administration remarkably maintained the paw withdrawal 330 latency in EODO treated rats.

$331 \quad$ Fig.3

332 LY294002 and Liriodendrin administration restored the up-regulated p-PI3K/p-AKT/p-mTOR level 333 in the endometrium of endometriosis rat models (* P value $<0.05$ vs. SHAM group; \# P value < 3340.05 vs. ENDO group).

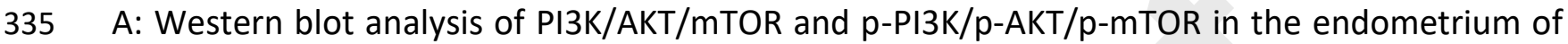
336 rats under distinct conditions.

337 B: Quantitative analysis indicated that no obvious difference was observed for the expression of $338 \mathrm{PI3K}$ in the endometrium of rats under distinct treatments.

339 C: Quantitative analysis indicated that no obvious difference was observed for the expression of 340 AKT in the endometrium of rats under distinct treatments.

341 D: Quantitative analysis indicated that no obvious difference was observed for the expression of 342 mTOR in the endometrium of rats under distinct treatments.

343 E: Quantitative analysis indicated LY294002 and Liriodendrin administration restored the up344 regulated $\mathrm{p}-\mathrm{PI3K}$ level in the ENDO group.

345 F: Quantitative analysis indicated LY294002 and Liriodendrin administration restored the up346 regulated $p$-AKT level in the ENDO group.

347 G: Quantitative analysis indicated LY294002 and Liriodendrin administration restored the up348 regulated $p$-mTOR level in the ENDO group.

$349 \quad$ Fig.4

350 Liriodendrin administration restored the elevation of IL-1, IL-2, IL-6, TNF- $\alpha$ and PGE2 in 351 endometriosis rat models (* P value < 0.05 vs. SHAM group; \# P value < 0.05 vs. ENDO group). 
352 A: Liriodendrin administration restored the elevated peritoneal fluid IL-1 level in the ENDO group.

353 B: Liriodendrin administration restored the elevated peritoneal fluid IL-2 level in the ENDO group.

354 C: Liriodendrin administration restored the elevated peritoneal fluid IL-6 level in the ENDO group.

355 D: Liriodendrin administration restored elevated peritoneal fluid TNF- $\alpha$ level in the ENDO group.

356 E: Liriodendrin administration restored elevated peritoneal fluid PGE2 level in the ENDO group.

357 F: Liriodendrin administration restored elevated serum PGE2 level in the ENDO group.

$358 \quad$ Fig. 5

359 Liriodendrin administration restored the increased CGRP and substance $P$ in the ventral horn of 360 spinal cord samples collected from rats with endometriosis (* P value $<0.05$ vs. SHAM group; \# $361 \quad P$ value $<0.05$ vs. ENDO group).

362 A: ELISA analysis indicated that Liriodendrin administration restored the elevated substance $P$ in 363 the ENDO rats.

364 B: ELISA analysis indicated that Liriodendrin administration restored the elevated CGRP in the 365 ENDO rats.

366 C: QPCR analysis indicated that Liriodendrin administration restored the elevated substance $\mathrm{P}$ in 367 the ENDO rats.

368 D: QPCR analysis indicated that Liriodendrin administration restored the elevated CGRP in the 369 ENDO rats.

370 E: QPCR analysis indicated that Liriodendrin administration restored the elevated NF-KB in the 371 ENDO rats.

372 F: Western blot analysis of CGRP and substance P expression in the ventral horn of spinal cord of 373 rats under distinct conditions.

374 G: ELISA analysis indicated that Liriodendrin administration restored the elevated substance P in 375 the ENDO rats. 
$376 \mathrm{H}$ : ELISA analysis indicated that Liriodendrin administration restored the elevated CGRP in the 377 ENDO rats.

$378 \quad$ Fig.6

379 LY294002 and Liriodendrin administration restored the LPS induced up-regulation of $p-P I 3 K / p-$ 380 AKT/p-mTOR in $12 Z$ cells (* P value < 0.05 vs. NC group; \# P value $<0.05$ vs. LPS group).

381 A: Western blot analysis of PI3K/AKT/mTOR and $p-\mathrm{PI} 3 \mathrm{~K} / \mathrm{p}-\mathrm{AKT} / \mathrm{p}-\mathrm{mTOR}$ in $12 \mathrm{Z}$ cells under distinct 382 conditions.

383 B: Quantitative analysis indicated that no obvious difference was observed for the expression of $384 \mathrm{PI3K}$ in $12 \mathrm{Z}$ cells under distinct treatments.

385 C: Quantitative analysis indicated that no obvious difference was observed for the expression of 386 AKT in $12 Z$ cells under distinct treatments.

387 D: Quantitative analysis indicated that no obvious difference was observed for the expression of 388 mTOR in $12 Z$ cells under distinct treatments.

389 E: Quantitative analysis indicated LY294002 and Liriodendrin administration restored the LPS 390 induced up-regulation of $\mathrm{p}$-PI3K in $12 Z$ cells.

391 F: Quantitative analysis indicated LY294002 and Liriodendrin administration restored the LPS 392 induced up-regulation of p-AKT in $12 Z$ cells.

393 G: Quantitative analysis indicated LY294002 and Liriodendrin administration restored the LPS 394 induced up-regulation of p-mTOR in $12 Z$ cells.

$395 \quad$ Fig.7

396 Liriodendrin administration restored the LPS induced up-regulation of IL-6, TNF- $\alpha$, and IL-1 $\beta$ 397 mRNA and protein expression in $12 Z$ cells (* P value $<0.05$ vs. NC group; \# P value < 0.05 vs. LPS 398 group).

399 A: Liriodendrin administration restored the LPS induced up-regulation of IL-6 mRNA expression 400 in $12 Z$ cells. 
B: Liriodendrin administration restored the LPS induced up-regulation of TNF- $\alpha$ mRNA expression in $12 Z$ cells.

C: Liriodendrin administration restored the LPS induced up-regulation of IL-1 $\beta$ mRNA expression 404 in $12 Z$ cells.

405

D: Liriodendrin administration restored the LPS induced up-regulation of NF-KB mRNA expression 406 in $12 Z$ cells.

407

E: ELISA analysis indicated that Liriodendrin administration restored the LPS induced upregulation of IL-6 protein expression in $12 Z$ cells.

409 F: Western blot analysis indicated that Liriodendrin administration restored the LPS induced up410 regulation of TNF- $\alpha$ protein expression in $12 Z$ cells.

411 G: Western blot analysis indicated that Liriodendrin administration restored the LPS induced up412 regulation of IL-1 $\beta$ protein expression in $12 Z$ cells.

\section{References}

4141 Sang Q, Sun D, Chen Z, Zhao W: NGF and PI3K/Akt signaling participate in the ventral 415

4172 Fraser IS: Recognising, understanding and managing endometriosis. J Hum Reprod Sci $418 \quad 2008 ; 1: 56-64$.

4193 Alvarez P, Giudice LC, Levine JD: Impact of surgical excision of lesions on pain in a rat $420 \quad$ model of endometriosis. Eur J Pain 2015;19:103-110.

4214 Baker GS, Parsons WR, Welch JS: Endometriosis within the sheath of the sciatic nerve. 422 Report of two patients with progressive paralysis. J Neurosurg 1966;25:652-655.

4235 Giamberardino MA, Tana C, Costantini R: Pain thresholds in women with chronic pelvic $424 \quad$ pain. Curr Opin Obstet Gynecol 2014;26:253-259.

4256 Lu PY, Ory SJ: Endometriosis: current management. Mayo Clin Proc 1995;70:453-463.

4267 Honda H, Barrueto FF, Gogusev J, Im DD, Morin PJ: Serial analysis of gene expression reveals differential expression between endometriosis and normal endometrium. 
Possible roles for AXL and SHC1 in the pathogenesis of endometriosis. Reprod Biol Endocrinol 2008;6:59.

8 Laudanski P, Szamatowicz J, Kowalczuk O, Kuzmicki M, Grabowicz M, Chyczewski L: Expression of selected tumor suppressor and oncogenes in endometrium of women with endometriosis. Hum Reprod 2009;24:1880-1890.

9 Zhang H, Zhao X, Liu S, Li J, Wen Z, Li M: 17betaE2 promotes cell proliferation in endometriosis by decreasing PTEN via NFkappaB-dependent pathway. Mol Cell Endocrinol 2010;317:31-43.

10 Banu SK, Lee J, Speights VO, Jr., Starzinski-Powitz A, Arosh JA: Selective inhibition of prostaglandin E2 receptors EP2 and EP4 induces apoptosis of human endometriotic cells through suppression of ERK1/2, AKT, NFkappaB, and beta-catenin pathways and activation of intrinsic apoptotic mechanisms. Mol Endocrinol 2009;23:1291-1305.

11 Velarde MC, Aghajanova L, Nezhat CR, Giudice LC: Increased mitogen-activated protein kinase kinase/extracellularly regulated kinase activity in human endometrial stromal fibroblasts of women with endometriosis reduces 3',5'-cyclic adenosine 5'monophosphate inhibition of cyclin D1. Endocrinology 2009;150:4701-4712.

12 Cao Y, Ye Q, Zhuang M, Xie S, Zhong R, Cui J, Zhou J, Zhu Y, Zhang T, Cao L: Ginsenoside Rg3 inhibits angiogenesis in a rat model of endometriosis through the VEGFR-2-mediated PI3K/Akt/mTOR signaling pathway. PLoS One 2017;12:e0186520.

13 Cao Y, Zhang TT, Xie SW, Zhu Y, Shu LD, Song ZL, Cao L, Dai DY: [Effects of Caulis Sargentodoxae Granule on expressions of vascular endothelial growth factor and its receptor-2 in rats with endometriosis]. Zhong Xi Yi Jie He Xue Bao 2009;7:360-365.

14 Feng C, Li BG, Gao XP, Qi HY, Zhang GL: A new triterpene and an antiarrhythmic liriodendrin from Pittosporum brevicalyx. Arch Pharm Res 2010;33:1927-1932.

15 Lami N, Kadota S, Kikuchi T, Momose Y: Constituents of the roots of Boerhaavia diffusa L. III. Identification of $\mathrm{Ca} 2+$ channel antagonistic compound from the methanol extract. Chem Pharm Bull (Tokyo) 1991;39:1551-1555.

16 Ran XK, Wang XT, Liu PP, Chi YX, Wang BJ, Dou DQ, Kang TG, Xiong W: Cytotoxic constituents from the leaves of Broussonetia papyrifera. Chin J Nat Med 2013;11:269-273. 
45717 Jung HJ, Park HJ, Kim RG, Shin KM, Ha J, Choi JW, Kim HJ, Lee YS, Lee KT: In vivo antiinflammatory and antinociceptive effects of liriodendrin isolated from the stem bark of Acanthopanax senticosus. Planta Med 2003;69:610-616.

18 Williams C, DiLeo A, Niv Y, Gustafsson JA: Estrogen receptor beta as target for colorectal cancer prevention. Cancer Lett 2016;372:48-56.

19 Liu W, Liu Y, Wang Z, Yu T, Lu Q, Chen H: Suppression of MAPK and NF-kappa B pathways

20 Tokuhira N, Kitagishi Y, Suzuki M, Minami A, Nakanishi A, Ono Y, Kobayashi K, Matsuda S, 466

21 Pages G, Pouyssegur J: Transcriptional regulation of the Vascular Endothelial Growth 469 Factor gene--a concert of activating factors. Cardiovasc Res 2005;65:564-573.

24 Fathi E, Vietor I: Mesenchymal Stem Cells Promote Caspase Expression in Molt-4 Leukemia

23 Fathi E, Farahzadi R, Vietor I, Javanmardi S: Cardiac differentiation of bone-marrow-resident c-kit+ stem cells by L-carnitine increases through secretion of VEGF, IL6, IGF-1, and TGF$\beta$ as clinical agents in cardiac regeneration. J Biosci, 2020;45:92.

23 Fathi E, Vietor I: Mesenchymal Stem Cells Promote Caspase Expression in Molt-4 Leukemia Cells Via GSK-3 $\alpha / B$ and ERK1/2 Signaling Pathways as a Therapeutic Strategy. Curr Gene Ther 2021;21(1):81-88.

48225 Fathi E, Farahzadi R, Javanmardi S, Vietor I. L-carnitine Extends the Telomere Length of the 
26 Fathi E, Farahzadi R, Valipour B: Alginate/gelatin encapsulation promotes NK cells differentiation potential of bone marrow resident C-kit ${ }^{+}$hematopoietic stem cells. Int J Biol Macromol 2021;177:317-327.

27 Ran XK, Wang XT, Liu PP, Chi YX, Wang BJ, Dou DQ, Kang TG, Xiong W: Cytotoxic constituents from the leaves of Broussonetia papyrifera. Chin J Nat Med 2013;11:269-273.

28 Zhang Z, Yang L, Wang B, Zhang L, Zhang Q, Li D, Zhang S, Gao H, Wang X. Protective role of liriodendrin in mice with dextran sulphate sodium-induced ulcerative colitis. Int Immunopharmacol, 2017;52:203-210.

29 Yang L, Li D, Zhuo Y, Zhang S, Wang X, Gao H. Protective Role of Liriodendrin in SepsisInduced Acute Lung Injury. Inflammation, 2016;39(5):1805-13.

30 Varma R, Rollason T, Gupta JK, Maher ER: Endometriosis and the neoplastic process. Reproduction 2004;127:293-304.

31 Somigliana E, Vigano P, Parazzini F, Stoppelli S, Giambattista E, Vercellini P: Association between endometriosis and cancer: a comprehensive review and a critical analysis of clinical and epidemiological evidence. Gynecol Oncol 2006;101:331-341.

32 Yagyu T, Tsuji Y, Haruta S, Kitanaka T, Yamada Y, Kawaguchi R, Kanayama S, Tanase Y, Kurita N, Kobayashi $\mathrm{H}$ : Activation of mammalian target of rapamycin in postmenopausal ovarian endometriosis. Int J Gynecol Cancer 2006;16:1545-1551.

33 Duan W, Aguinaldo Datiles AM, Leung BP, Vlahos CJ, Wong WS: An anti-inflammatory role for a phosphoinositide 3-kinase inhibitor LY294002 in a mouse asthma model. Int Immunopharmacol 2005;5:495-502.

34 Kwak YG, Song CH, Yi HK, Hwang PH, Kim JS, Lee KS, Lee YC: Involvement of PTEN in airway hyperresponsiveness and inflammation in bronchial asthma. J Clin Invest 2003;111:10831092.

35 Takeshima E, Tomimori K, Kawakami H, Ishikawa C, Sawada S, Tomita M, Senba M, Kinjo F, Mimuro H, Sasakawa C, Fujita J, Mori N: NF-kappaB activation by Helicobacter pylori requires Akt-mediated phosphorylation of p65. BMC Microbiol 2009;9:36. 
51136 Kim DH, Lee KT, Bae EA, Han MJ, Park HJ: Metabolism of liriodendrin and syringin by 512 human intestinal bacteria and their relation to in vitro cytotoxicity. Arch Pharm Res 513 1999;22:30-34.

51437 Cho S, Cho M, Kim J, Kaeberlein M, Lee SJ, Suh Y: Syringaresinol protects against 515 hypoxia/reoxygenation-induced cardiomyocytes injury and death by destabilization of 516 HIF-1alpha in a FOXO3-dependent mechanism. Oncotarget 2015;6:43-55.

51738 Park HW, Cho SY, Kim HH, Yun BS, Kim JU, Lee SJ, Park J: Enantioselective induction of 518 SIRT1 gene by syringaresinol from Panax ginseng berry and Acanthopanax senticosus 519 Harms stem. Bioorg Med Chem Lett 2015;25:307-309. 

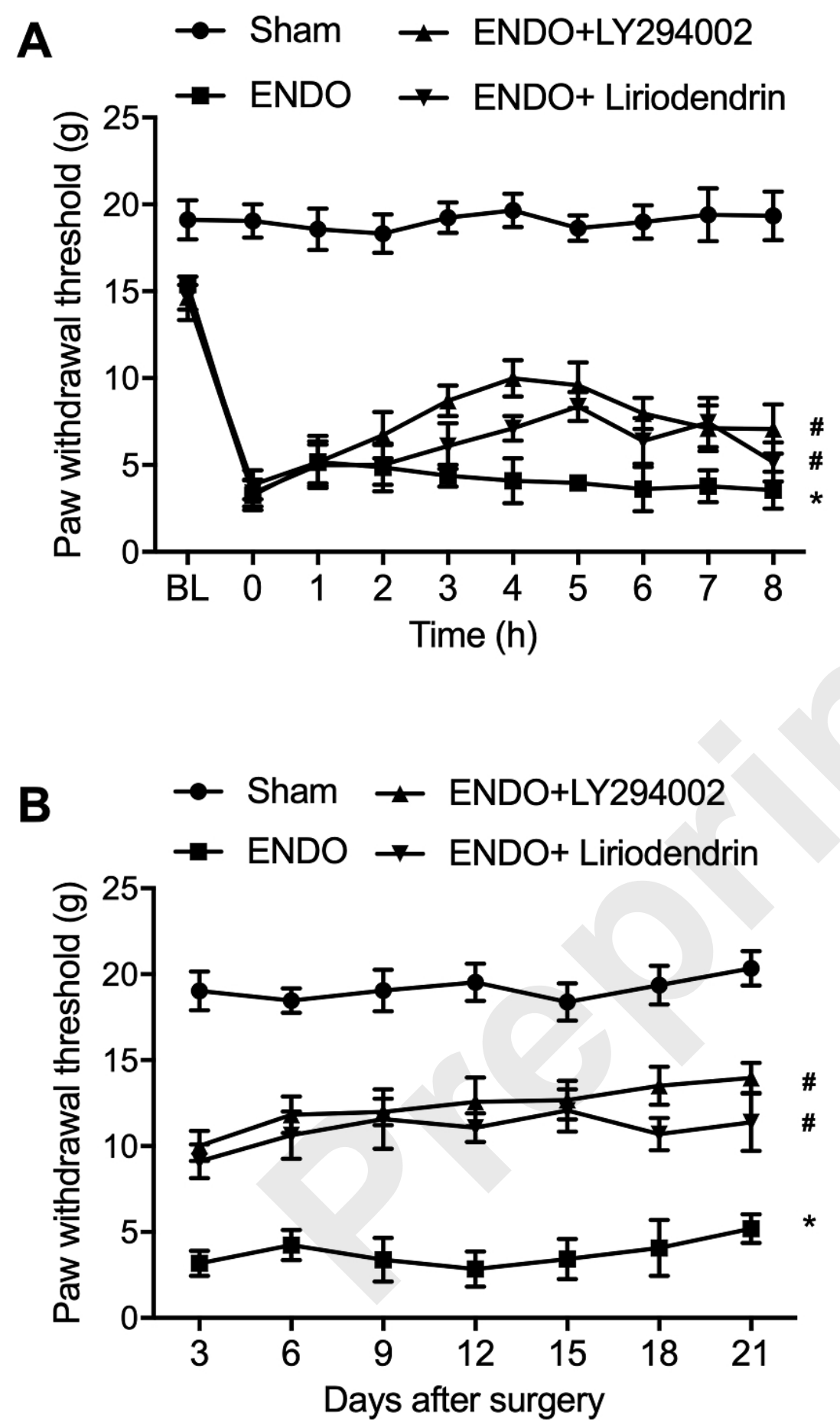

Fig. 1

Chemical structure of Liriodendrin and the efficiency of acute/daily drug administration evaluated by Von Frey filament test ( ${ }^{*}$ value $<0.05$ vs. SHAM group; \# P value $<0.05$ vs. ENDO group). A: Acute LY294002 and Liriodendrin administration remarkably maintained the paw withdrawal threshold in EODO treated rats.

B: Daily LY294002 and Liriodendrin administration remarkably maintained the paw withdrawal threshold in EODO treated rats. 

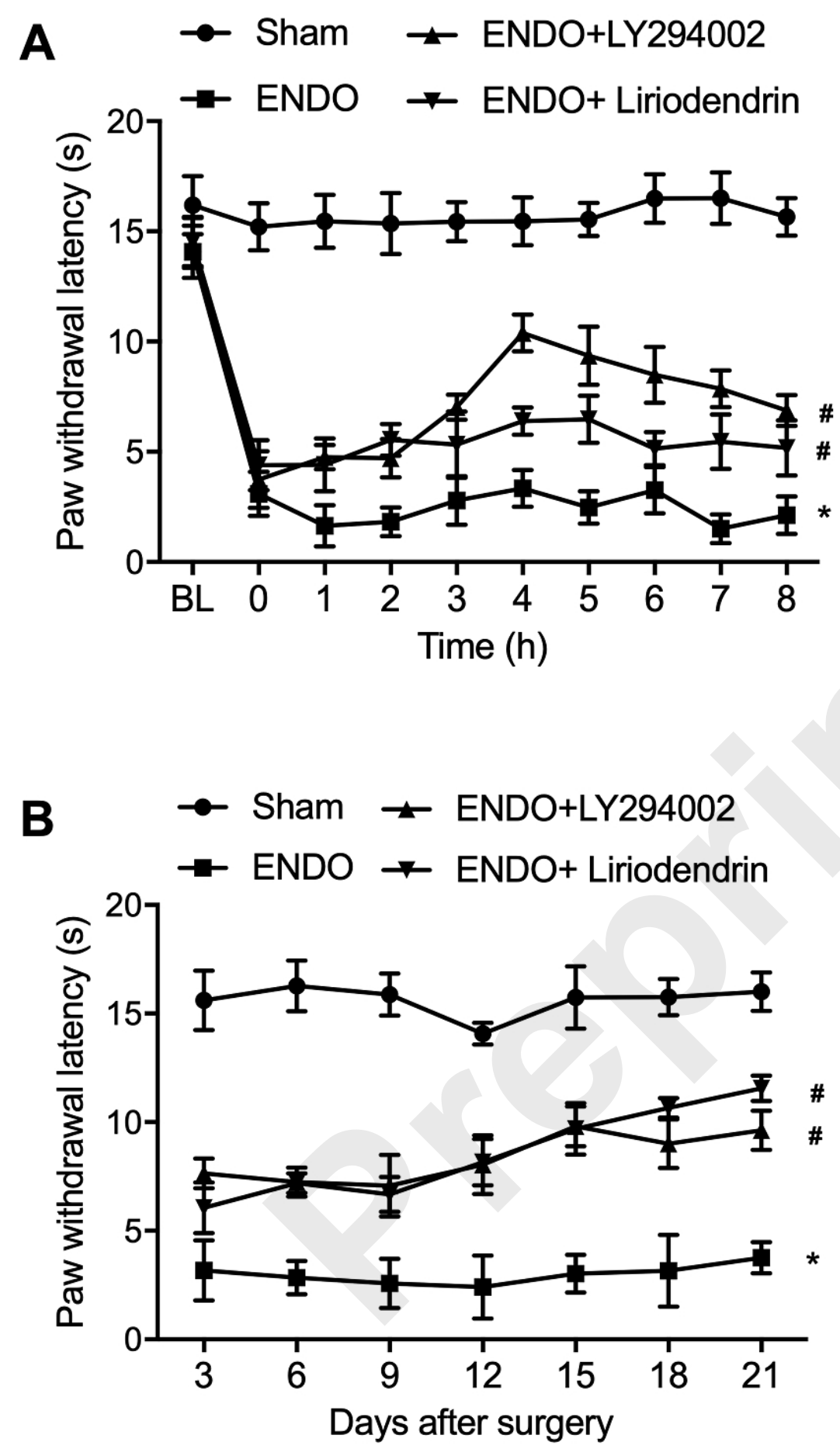

Fig.2

Acute/daily drug administration efficiency evaluation using Thermal hyperalgesia test ( $\mathrm{P}$ value $<0.05$ vs. SHAM group; \# P value $<0.05$ vs. ENDO group).

A: Acute LY294002 and Liriodendrin administration remarkably maintained the paw withdrawal latency in EODO treated rats.

B: Daily LY294002 and Liriodendrin administration remarkably maintained the paw withdrawal latency in EODO treated rats. 


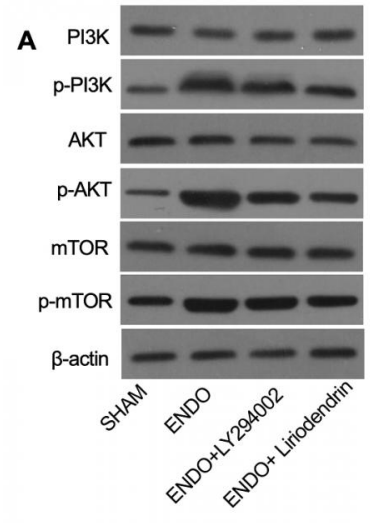

E

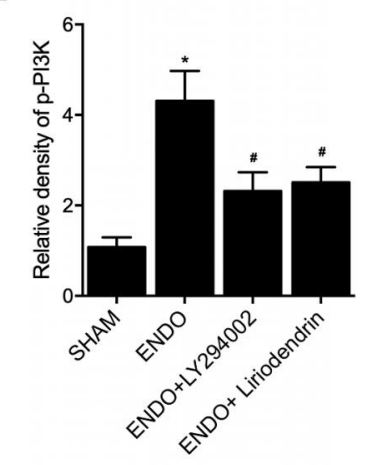

B

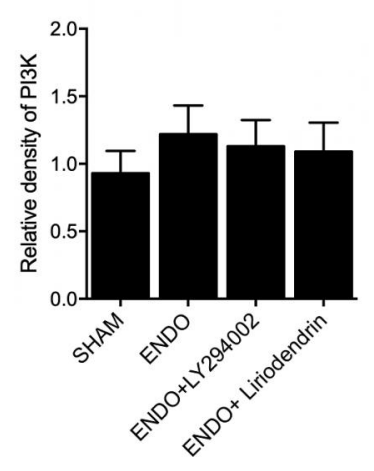

$\mathbf{F}$

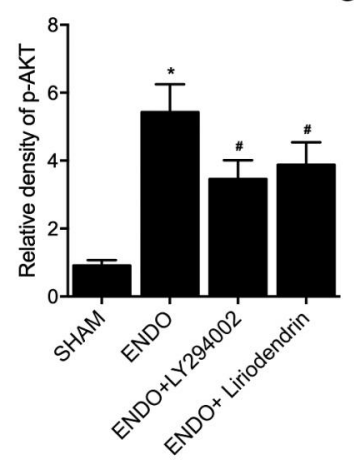

C

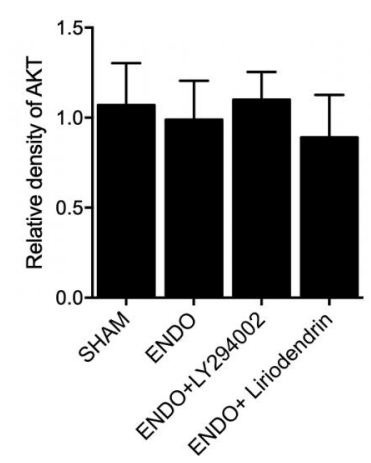

G

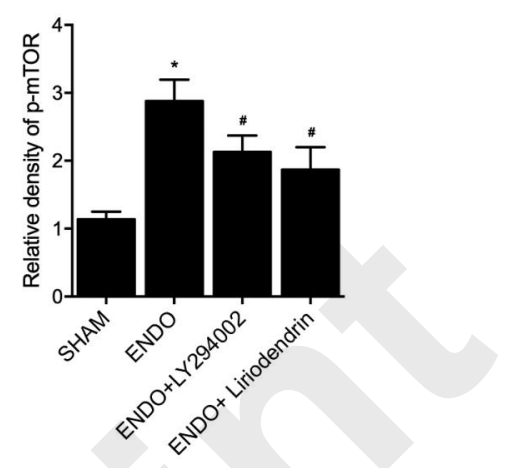

Fig.3

LY294002 and Liriodendrin administration restored the up-regulated p-PI3K/p-AKT/p-mTOR level in the endometrium of endometriosis rat models ( ${ }^{*} P$ value $<0.05$ vs. SHAM group; \# $P$ value $<0.05$ vs. ENDO group).

A: Western blot analysis of PI3K/AKT/mTOR and p-PI3K/p-AKT/p-mTOR in the endometrium of rats under distinct conditions.

B: Quantitative analysis indicated that no obvious difference was observed for the expression of $\mathrm{PI} 3 \mathrm{~K}$ in the endometrium of rats under distinct treatments.

C: Quantitative analysis indicated that no obvious difference was observed for the expression of AKT in the endometrium of rats under distinct treatments.

D: Quantitative analysis indicated that no obvious difference was observed for the expression of $\mathrm{mTOR}$ in the endometrium of rats under distinct treatments.

E: Quantitative analysis indicated LY294002 and Liriodendrin administration restored the upregulated $\mathrm{p}-\mathrm{PI} 3 \mathrm{~K}$ level in the ENDO group.

F: Quantitative analysis indicated LY294002 and Liriodendrin administration restored the upregulated p-AKT level in the ENDO group.

G: Quantitative analysis indicated LY294002 and Liriodendrin administration restored the upregulated p-mTOR level in the ENDO group. 
A
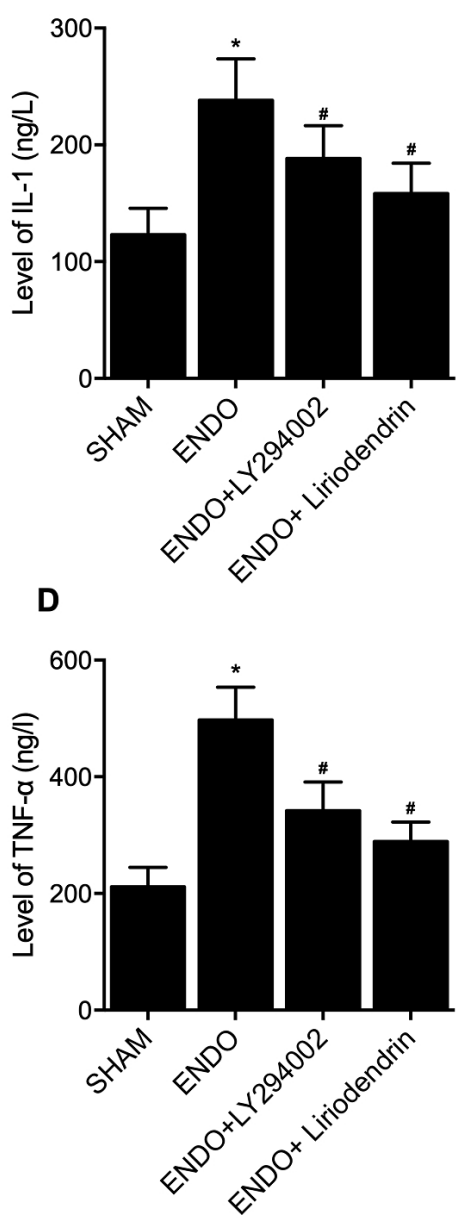

B

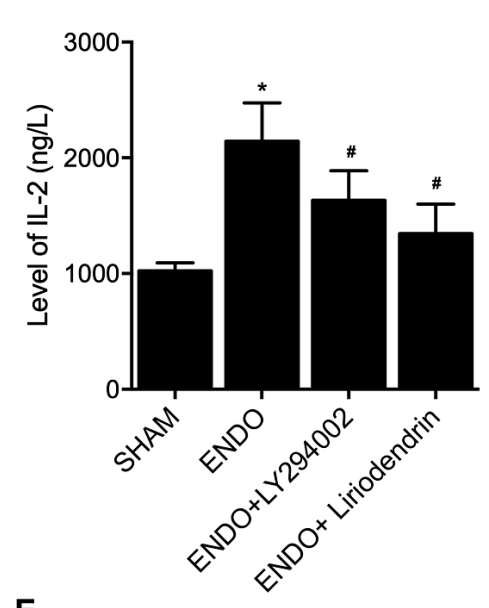

$\mathbf{E}$

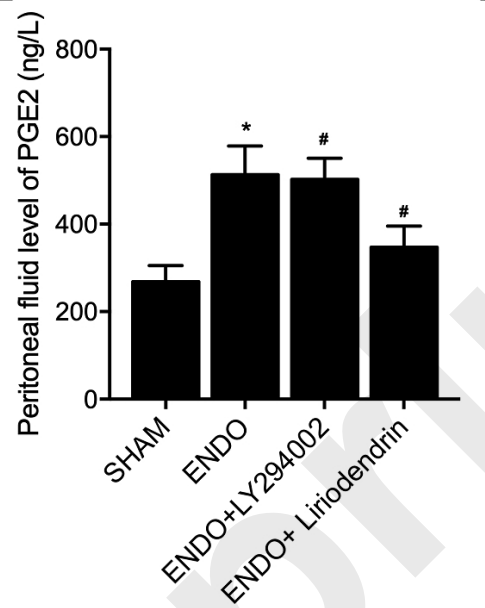

C
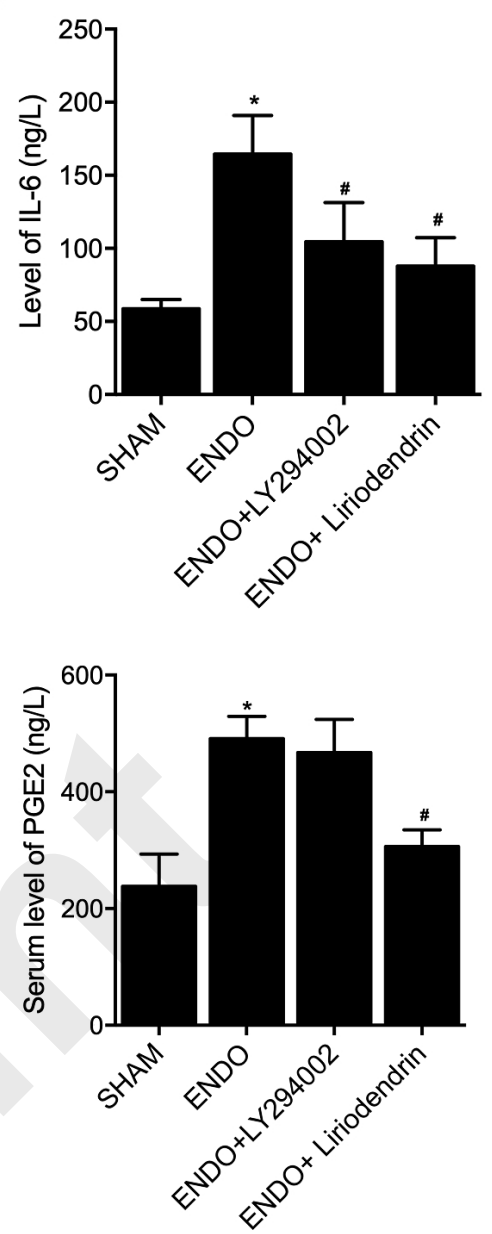

Fig.4

Liriodendrin administration restored the elevation of IL-1, IL-2, IL-6, TNF- $\alpha$ and PGE2 in endometriosis rat models ( ${ }^{*} P$ value $<0.05$ vs. SHAM group; \# $P$ value $<0.05$ vs. ENDO group).

A: Liriodendrin administration restored the elevated peritoneal fluid IL-1 level in the ENDO group.

B: Liriodendrin administration restored the elevated peritoneal fluid IL-2 level in the ENDO group.

C: Liriodendrin administration restored the elevated peritoneal fluid IL-6 level in the ENDO group.

D: Liriodendrin administration restored elevated peritoneal fluid TNF- $\alpha$ level in the ENDO group.

E: Liriodendrin administration restored elevated peritoneal fluid PGE2 level in the ENDO group.

F: Liriodendrin administration restored elevated serum PGE2 level in the ENDO group. 
A

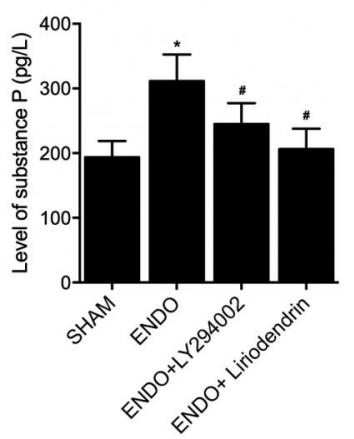

E

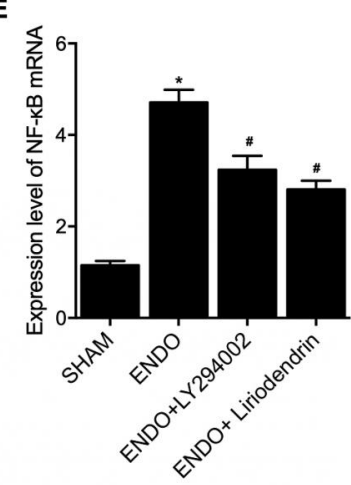

B

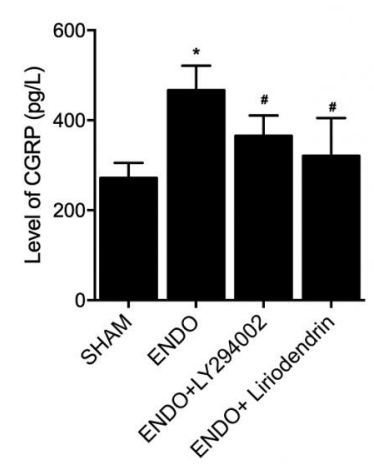

$\mathbf{F}$

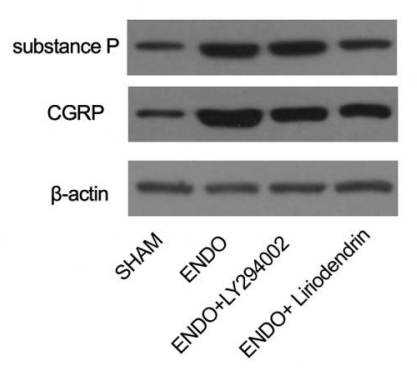

C

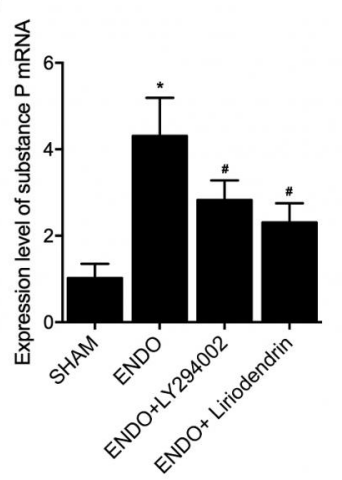

G

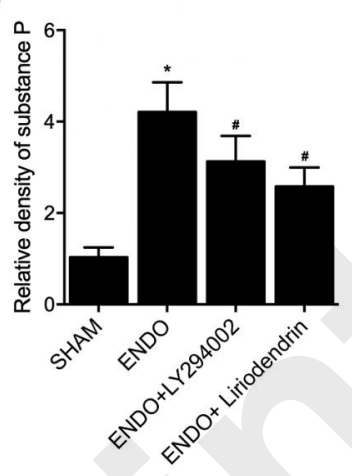

D

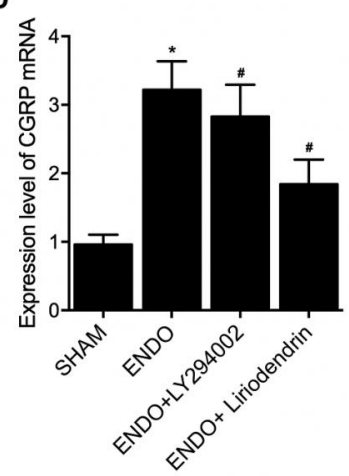

H

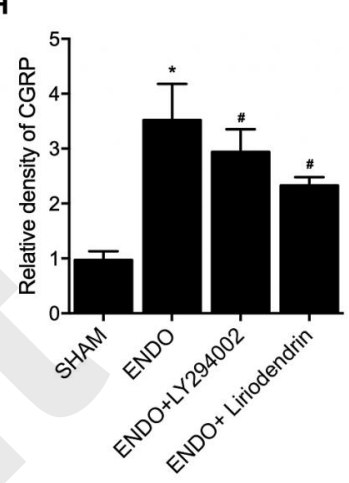

Fig.5

Liriodendrin administration restored the increased CGRP and substance $P$ in the ventral horn of spinal cord samples collected from rats with endometriosis ( ${ }^{*} P$ value $<0.05$ vs.

SHAM group; \# P value < 0.05 vs. ENDO group).

A: ELISA analysis indicated that Liriodendrin administration restored the elevated substance $P$ in the ENDO rats.

$\mathrm{B}$ : ELISA analysis indicated that Liriodendrin administration restored the elevated CGRP in the ENDO rats.

C: QPCR analysis indicated that Liriodendrin administration restored the elevated substance $P$ in the ENDO rats.

$\mathrm{D}$ : QPCR analysis indicated that Liriodendrin administration restored the elevated CGRP in the ENDO rats.

$\mathrm{E}: \mathrm{QPCR}$ analysis indicated that Liriodendrin administration restored the elevated NF-KB in the ENDO rats.

$\mathrm{F}$ : Western blot analysis of CGRP and substance P expression in the ventral horn of spinal cord of rats under distinct conditions.

G: ELISA analysis indicated that Liriodendrin administration restored the elevated substance $P$ in the ENDO rats.

$\mathrm{H}$ : ELISA analysis indicated that Liriodendrin administration restored the elevated CGRP in the ENDO rats. 


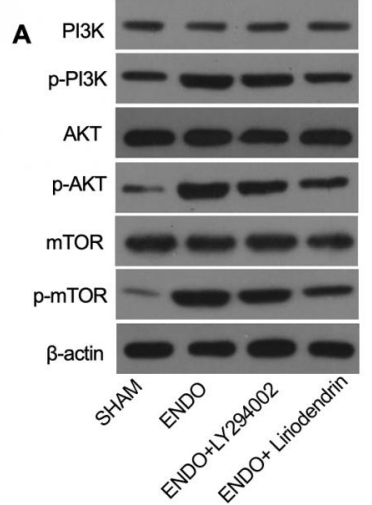

E

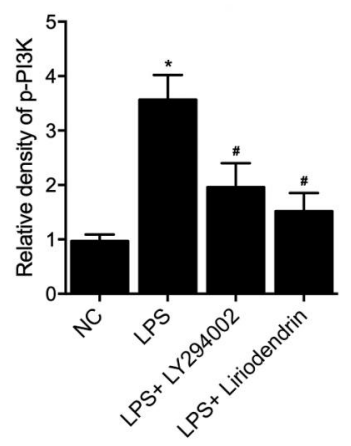

B
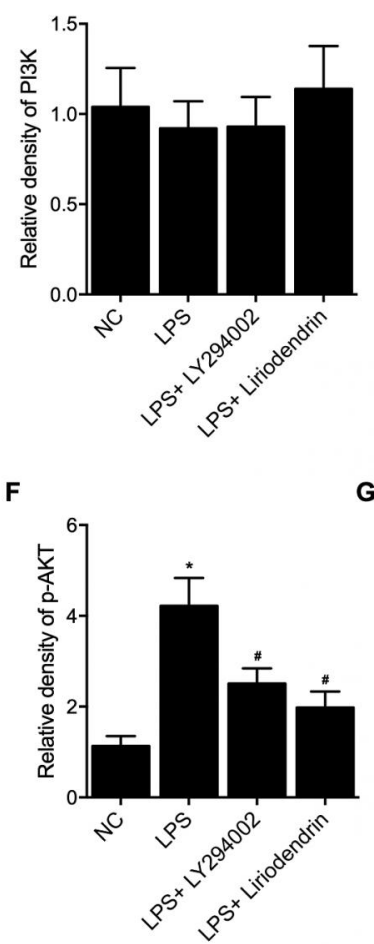

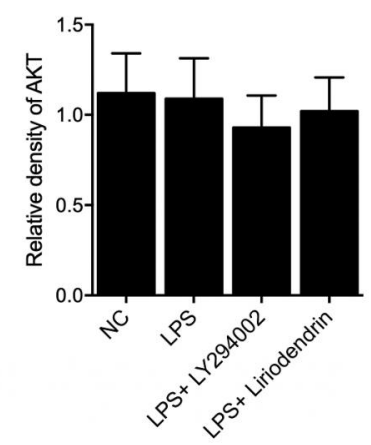

G

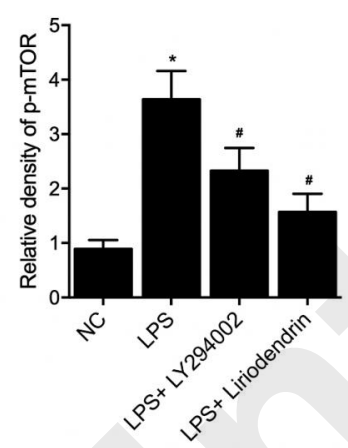

D

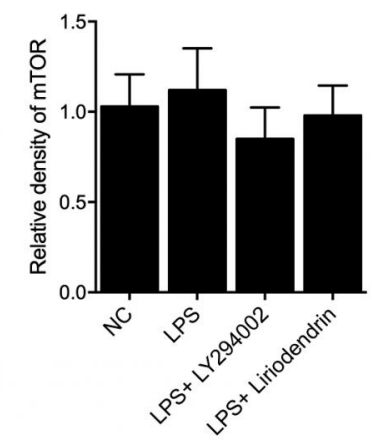

Fig.6

LY294002 and Liriodendrin administration restored the LPS induced up-regulation of p$\mathrm{PI3K} / \mathrm{p}-\mathrm{AKT} / \mathrm{p}-\mathrm{mTOR}$ in $12 \mathrm{Z}$ cells $\left({ }^{*} \mathrm{P}\right.$ value $<0.05$ vs. NC group; \# $\mathrm{P}$ value $<0.05$ vs. LPS group).

A: Western blot analysis of PI3K/AKT/mTOR and p-PI3K/p-AKT/p-mTOR in $12 Z$ cells under distinct conditions.

B: Quantitative analysis indicated that no obvious difference was observed for the expression of $\mathrm{PI} 3 \mathrm{~K}$ in $12 \mathrm{Z}$ cells under distinct treatments.

C: Quantitative analysis indicated that no obvious difference was observed for the expression of AKT in $12 Z$ cells under distinct treatments.

D: Quantitative analysis indicated that no obvious difference was observed for the expression of mTOR in $12 Z$ cells under distinct treatments.

E: Quantitative analysis indicated LY294002 and Liriodendrin administration restored the LPS induced up-regulation of $\mathrm{p}-\mathrm{PI} 3 \mathrm{~K}$ in $12 \mathrm{Z}$ cells.

F: Quantitative analysis indicated LY294002 and Liriodendrin administration restored the LPS induced up-regulation of p-AKT in $12 Z$ cells.

G: Quantitative analysis indicated LY294002 and Liriodendrin administration restored the LPS induced up-regulation of $p-m T O R$ in $12 Z$ cells. 

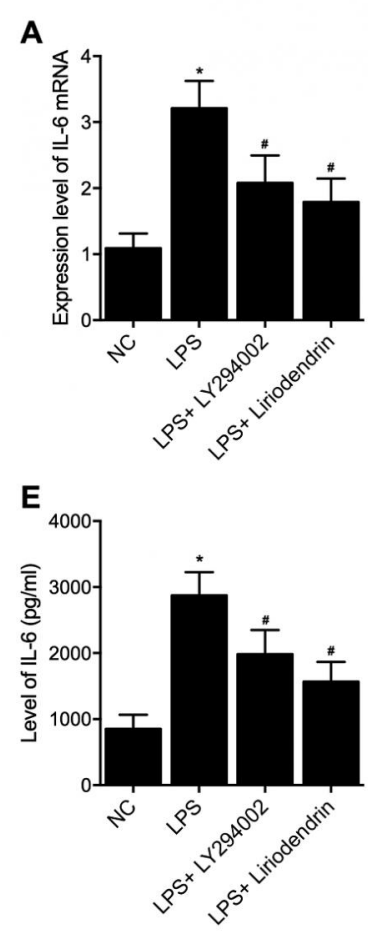

B

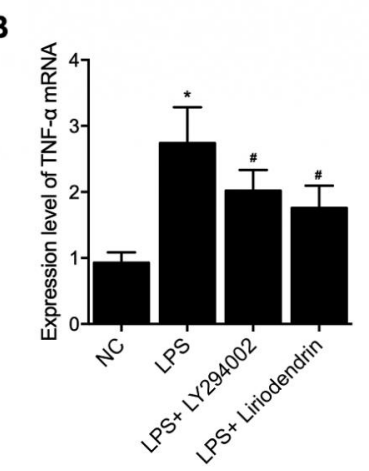

$\mathbf{F}$

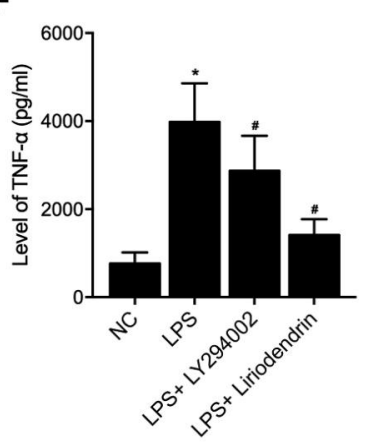

C

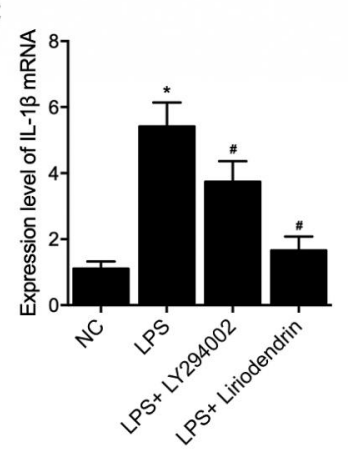

G

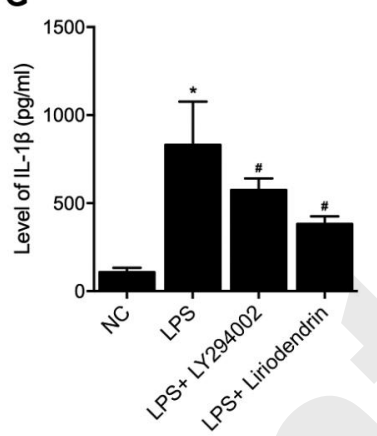

D

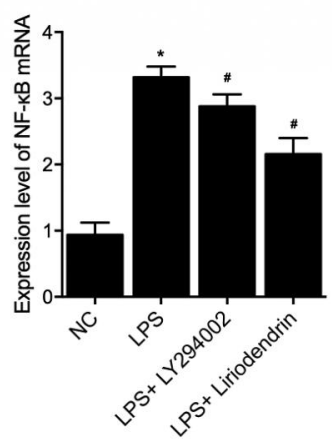

Fig.7

Liriodendrin administration restored the LPS induced up-regulation of IL-6, TNF- $\alpha$, and $\mathrm{IL}-1 \beta$ mRNA and protein expression in $12 \mathrm{Z}$ cells ( ${ }^{*} \mathrm{P}$ value $<0.05$ vs. NC group; \# $\mathrm{P}$ value $<$ 0.05 vs. LPS group).

A: Liriodendrin administration restored the LPS induced up-regulation of IL-6 mRNA expression in $12 Z$ cells.

$B$ : Liriodendrin administration restored the LPS induced up-regulation of TNF- $\alpha$ mRNA expression in $12 Z$ cells.

C: Liriodendrin administration restored the LPS induced up-regulation of IL-1 $\beta$ mRNA expression in $12 Z$ cells.

$\mathrm{D}$ : Liriodendrin administration restored the LPS induced up-regulation of NF-kB mRNA expression in $12 Z$ cells.

E: ELISA analysis indicated that Liriodendrin administration restored the LPS induced upregulation of IL-6 protein expression in $12 Z$ cells.

$\mathrm{F}$ : Western blot analysis indicated that Liriodendrin administration restored the LPS induced up-regulation of TNF- $\alpha$ protein expression in $12 Z$ cells.

G: Western blot analysis indicated that Liriodendrin administration restored the LPS induced up-regulation of IL-1 $\beta$ protein expression in $12 Z$ cells. 